\title{
Comparison of two points of view when developing reliability-based topology optimization model: Validation on fatigue damage analysis
}

\section{Comparaison de deux points de vue lors de l'élaboration d'un modèle d'optimisation fiabiliste de topologie : Validation sur l'analyse de} l'endommagement par fatigue

Kharmanda G. ${ }^{1 *}$; Shokry A. ${ }^{2}$; Gowid S. ${ }^{3}$; El-Hami A. ${ }^{1}$

\footnotetext{
${ }^{1}$ Mechanics Laboratory of Normandy, INSA Rouen, France

${ }^{2}$ Department of Mechanical Engineering, Fayoum University, Egypt, abdallah.shokry@fayoum.edu.eg

${ }^{3}$ Department of Mechanical and Industrial Engineering, Qatar University, Qatar, samer@qu.edu.qa

*Corresponding author: mgk@scs-net.org
}

ABSTRACT. The classical topology optimization leads to a prediction of the structural type and overall layout configuration, and gives a rough shape description of the outer as well as inner boundaries of the structure. While, the Reliability-Based Topology Optimization (RBTO) model leads to several reliability-based topologies with high performance levels. Several strategies have been developed in this area considering two points of view: topology optimization and reliability analysis. In this work, a literature review of this different developments considering the two points of view over the last 20 years is presented to show that the different developments considering the point of view 'topology optimization' generate several reliability-based topologies, however, the other developments considering the point of view 'reliability analysis' lead to a single configuration. A numerical application on fatigue damage analysis is considered as a validation of the developed methods taking the point of view 'topology optimization' into account.

KEYWORDS. Deterministic Topology Optimization (DTO), Reliability-Based Topology Optimization, Reliability Analysis, Gradient-Based Method (GBM), Fatigue Damage Analysis.

\section{Introduction}

Topology optimization can answer one of the first questions concerning the nature of the structure to fulfill the necessary technical specifications. The topology problem consists of determining the structure's general characteristics. The goal of topology optimization is to make that initial choice as automatically as possible (Bendsøe and Kikuchi 1988). In addition, when using topology optimization concepts, both macroscopic structures and microscopic materials can be found. In other word, the optimal spatial material layout distribution at the macroscopic structural scale and the optimal local use of the cellular material at the microscopic scale are determined (Xia 2016).

In the literature, two main models of topology optimization can be distinguished: deterministic model and reliability-based model. In Deterministic Topology Optimization (DTO), a single solution for a given domain can be found (Zhang et al. 2016). In contrast, the Reliability-Based Topology Optimization (RBTO) model can lead to several configurations with different advantages. This way, the best solution to fulfill the technical specifications can be chosen. The structural weight of the resulting topologies obtained by this model is reduced when comparing with the DTO model. Furthermore, when using the RBTO model, the resulting structure is found to be more reliable when comparing with the deterministic topology for the same weight levels (Kharmanda and El-Hami 
2017). Two points of view are presented for the development of this model: topology optimization and reliability analysis. A literature review is established in the next section, to show the different advantages of the RBTO model considering the two points of view.

\section{Actual developments considering two points of view}

The main difference between deterministic topology optimization and reliability-based topology optimization is to consider the uncertainty on the parameters having important roles for optimal topology. The main idea of the RBTO is based on the concept of the Reliability-Based Design Optimization (RBDO). In RBDO problem, the uncertainties regarding the sizing variables are taken into account in order to improve the reliability of the proposed solution. However, the RBTO seeks to provide designers with several configurations that have several reliability levels. This way the designer can choose the best solution. In the RBTO model, several methods have been developed, in which the different developments can be classified into two points of view:

From point of view 'topology optimization', Kharmanda and Olhoff (2001) have elaborated an RBTO model with object of providing the designer with several reliability-based configurations, while in the deterministic topology optimization, the designer produces only one deterministic topology. It has been shown the RBTO model yields structures that should be more reliable than those produced by deterministic topology optimization for the same weight (Kharmanda and Olhoff 2002; Kharmanda 2003; Kharmanda et al. 2004; Kharmanda et al. 2007b; Kharmanda and AlKhatab 2011). In order to validate the developed algorithm using Gradient-Based Method (GBM), a comparison of the resulting topologies of the developed algorithm with ANSYS software have been carried out (Kharmanda et al. 2007a; Kharmanda and Al-Bashi 2011). This approach has been extended to biomechanical applications (Kharmanda and Ibrahim 2013; Kharmanda and Al-Bashi 2013; Kharmanda and El-Hami 2017). In the RBTO model, the reliability constraints have been introduced into deterministic topology optimization formulation. The initial sensitivity analysis is used to identify random variables which have significant effect on the objective function and the limit state function is considered as a linear combination of the random variables. Therefore, the proposed approach is considered as a heuristic strategy that aims to reduce mass while improving the reliability level of the structure without greatly increasing its weight. But the limit state function used by them was not based on failure criteria for the structure, which can be treated in a different way comparing to the RBDO model. The developed formulation considered uncertainty with respect to geometrical dimension and applied loading. In addition, their reliability analysis seems to be independent of the boundary and loading condition. So their results showed similar values for the uncertain variables for different structures. Next, Patel and Choi (2012) used probabilistic neural networks in the case of highly nonlinear or disjoint failure domain problems. Their strategy has been efficiently applied on different truss structures. After that, Wang et al. (2017) developed a nonprobabilistic reliability-based topology optimization method for detailed design of continuum structures, in which the Unknown But Bounded uncertainties (UBB) existing in material and external loads are considered simultaneously. Their results show that the consideration of the UBB uncertainty effects during the whole procedure of topology optimization may have a significant influence on the final structural layout. Recently, Kharmanda et al. (2019a) and (2019b) developed an efficient tool, called Inverse Optimum Safety (IOSF) method, which is applied to modal analysis cases. The effect of the reliability index values on the resulting reliability-based topology configurations is also studied by Kharmanda et al. (2019c).

From a point of view 'reliability analysis', the deterministic topology optimization is formulated as finding the stiffest structural configuration with a volume constraint, where the feasibility of volume constraint is not critical in structural design problems. It seems to be more important to consider the variations of the stiffness under uncertainties. In order to maintain the robustness of 
stiffness in the topology design, Bae and Wang (2002) formulated the topology design optimization as volume minimization problem with a displacement constraint and their problem is solved using the RBDO technique. Jung et al. (2003) extended the work of Bae and Wang to non linear problems. Next, the Hybrid Cellular Automaton (HCA) method has been developed by Tovar et al. (2004) for structural synthesis of continuum material where the state of each cell is defined by both density and strain energy. In Agarwal (2004), a decoupled RBDO approach has been employed such that the topology optimization was separated from the reliability analysis. Patel et al. (2005) developed the RBTO model using the gradient free Hybrid Cellular Automata (HCA) method. Their formulation also incorporates uncertainty with respect to material property. They considered limit state function based on failure scenarios on the output displacements. Eom et al. (2011) carried out the RBTO model using bi-directional evolutionary structural optimization and the standard response surface method. A computational method for reliability-based topology optimization for continuum domains under material properties uncertainty was developed by Jalalpour and Tootkaboni (2016).

When comparing both different points of view, the RBTO methods considering the point of view 'reliability analysis' need a high computing time due to the additional required system analysis associate with RBDO since a large number of design variables are associated with continuum topology optimization problems. These methods lead to the same layout structures with different densities that have no sense for the following optimization stages.

However, the different methods considering the a point of view 'topology optimization' lead to interesting layouts for topology designers because they provide several reliability-based structures relative the reliability index changes.

\section{Reliability analysis}

To estimate the reliability levels, several techniques have been developed during the last 50 years such as: First Order Reliability Methods (FORM), Second Order Reliability Methods (SORM) and simulation techniques (Kharmanda and El-Hami 2016; Melchers and Beck 2017). In this work, the method developed by Hasofer and Lind (1974) is used. The image of the random variables in the standard normalized space is denoted $\mathbf{u}$, calculated by: $\mathbf{u}=T(\mathbf{x}, \mathbf{y})$ where $T(\mathbf{x}, \mathbf{y})$ is the probabilistic transformation function (see Fig. 1). For a given failure scenario, the reliability index $\beta$ is assessed by solving a constrained minimization problem:

$$
\beta=\min d(\mathbf{u}) \quad \text { s.t.: } \quad H(\mathbf{u}) \leq 0
$$

with

$$
d=\sqrt{\sum u_{i}^{2}}
$$

where $\mathbf{u}$ is the normalized vector, measured from the origin (see Fig. 1b). The solution to problem (1) defines the Most Probable failure Point (MPP). The minimum distance between the limit state function $H(\mathbf{u})=0$ and the origin, is called the reliability index $\beta$ (Hasofer and Lind 1974). 


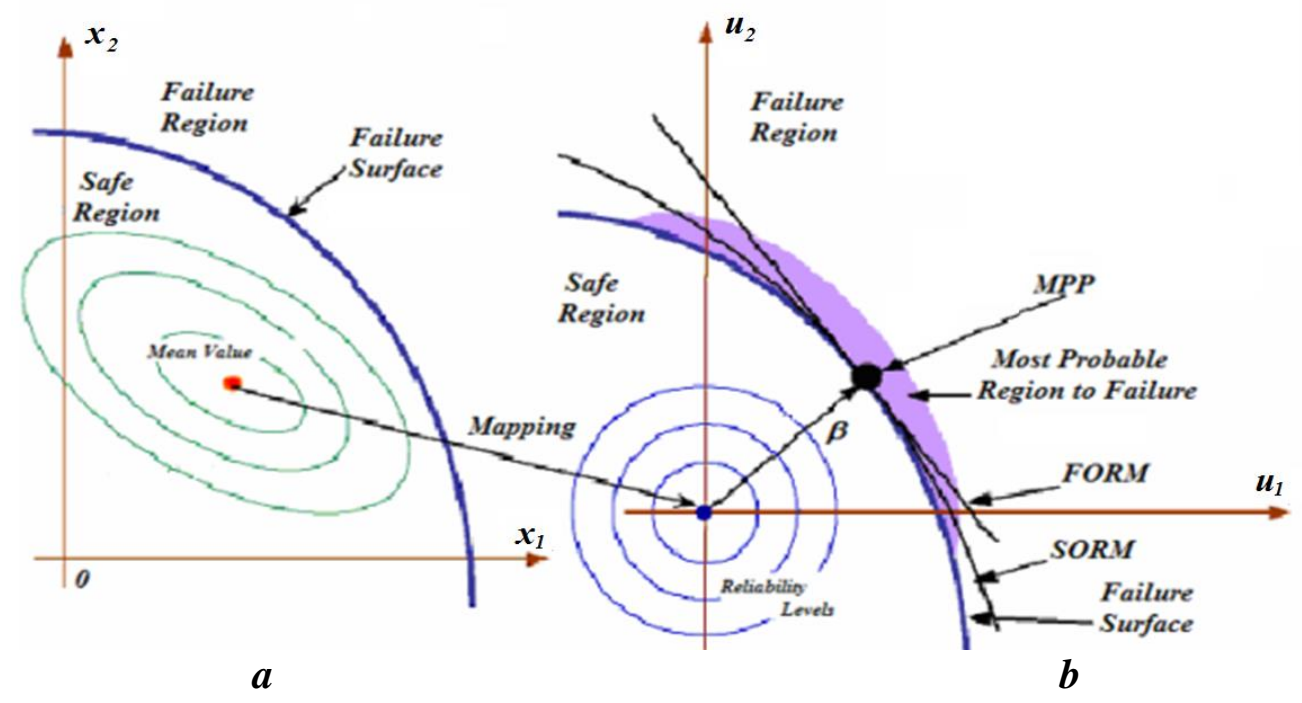

Fig. 1. Transformation between physical and normalized spaces.

\section{DTO versus RBTO}

The topology optimization aims to determine a sub-domain occupied by the constitutive material of a body, with no a priori knowledge of its topology - i.e. of the nature and connectivity of the elements making it up. The search for the structural domain is performed within a larger reference domain (initial domain). There are several topology optimization methods in the existing literature, which are capable of solving the topology problem. The general formulation of the DTO problem is:

$$
\begin{array}{ll}
\min & : f(\mathbf{x}) \\
\text { s.t. } & : g(\mathbf{x})=0
\end{array}
$$

The interest of topology optimization is very big from the point of view of the material savings that can be made whilst preserving good structural strength. The RBTO model improves the performance of the resulting reliability-based topologies in order to meet the different market requirements. The general formulation of the RBTO problem is:

$$
\begin{array}{ll}
\min & : f(\mathbf{x}) \\
\text { s.t. } & : g(\mathbf{x})=0 \\
\text { and } & : \beta(\mathbf{u}) \geq \beta
\end{array}
$$

Here, the reliability constraints are added to the optimization problem in order to provide a different layout.

\section{Application on fatigue damage analysis}

Consider an MBB beam subjected to a multi-axial stress due to the action of the distributed loads (Fig. 2) and the required (target) reliability index equals to: $\beta_{t}=3.8$. In all cases of this study, the number of elements in the horizontal and vertical directions are: nelx $=80$ and nely $=16$, respectively. The initial volume of the beam is then: $V_{0}=1280 \mathrm{~mm}^{3}$. By integrating reliability 
constraint $\beta(\mathbf{u}) \geq \beta_{t}$, the random vector $\mathbf{y}$ is obtained and the initial volume corresponding to the RBTO model is: $V_{0}=1960 \mathrm{~mm}^{3}$.

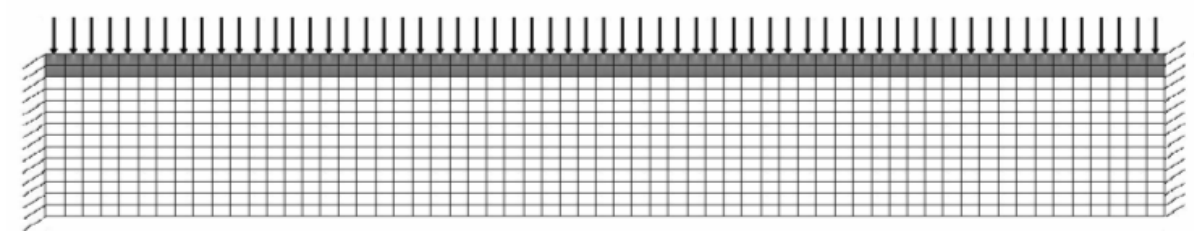

Fig. 2. MBB beam subjected to a multi-axial stress due to the action of distributed loads

\section{Results of DTO and RBTO considering the point of view of 'topology optimization'}

\section{Case 1: Minimization of volume subject to a constraint of fatigue damage}

The DTO problem is to minimize the structural volume subjected to a constraint of fatigue damage. It can be written as follows:

$$
\begin{aligned}
& \min : \operatorname{Volume}(x) \\
& \text { s.t. }: \max \mathbf{D} \leq \mathrm{D}_{U}
\end{aligned}
$$

$D_{U}=0.53$ is the maximum fatigue damage (Lambert et al. 2007). The deterministic topology illustrated in Fig. 3a, satisfies a minimal volume and the maximum damage value Dmax = $0.525 \approx D U$. The RBTO problem can be written as:

$$
\begin{aligned}
& \min : \operatorname{Volume}(x) \\
& \text { s.t. }: \max \mathbf{D} \leq \mathrm{D}_{U} \\
& \text { and }: \beta(\mathbf{u}) \geq \beta_{t}
\end{aligned}
$$

The resulting reliability-based topology illustrated in Fig. $3 b$ gives a minimal volume which is larger than that obtained by DTO, and the maximal damage Dmax $=0.52 \approx D U$ satisfies the target reliability level.
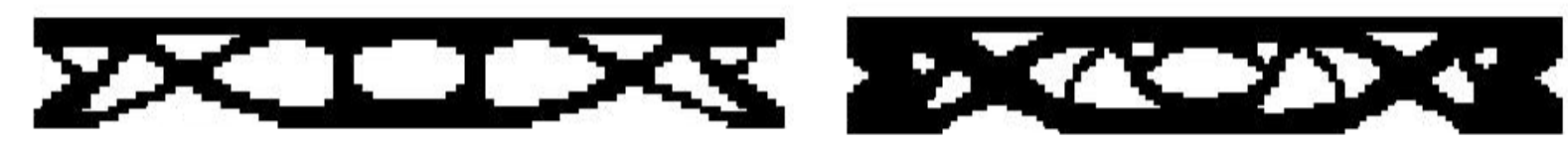

$a$
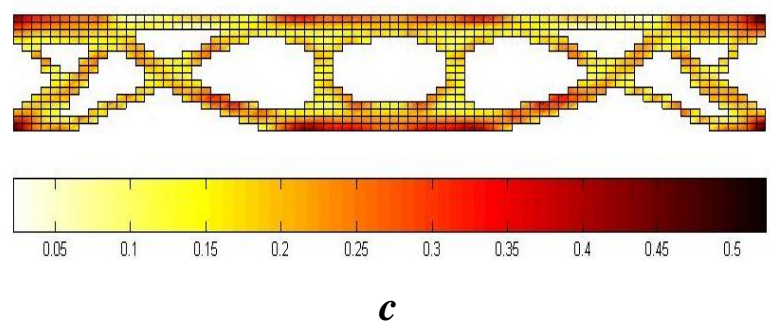
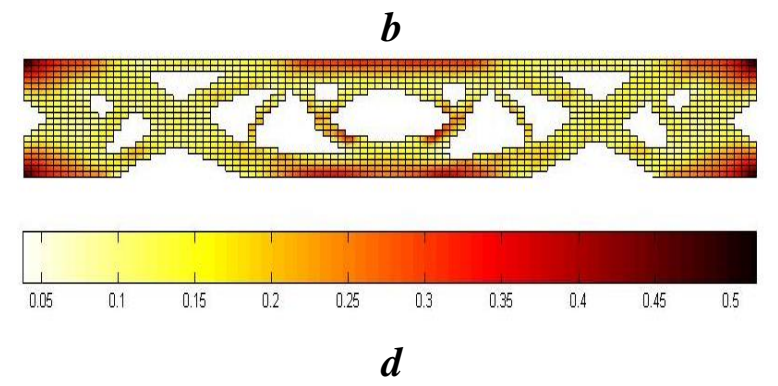

Fig. 3. Topologies and damage distributions: a,c) DTO and b,d) RBTO models

Figs. 3a, b, c and d show the resulting topologies and their damage distributions for the DTO model ( $3 a$ and $c$ ) and for the RBTO model ( $3 b$ and $d)$. Table 1 presents the DTO and RBTO results for problems 5 and 6. 


\begin{tabular}{|c|c|c|c|c|c|c|c|}
\hline \multirow{2}{*}{ Model } & \multirow{2}{*}{$\begin{array}{c}\text { Input } \\
\text { vector }\end{array}$} & \multicolumn{4}{|c|}{ Input parameters } & \multicolumn{2}{c|}{ Results } \\
\cline { 3 - 8 } & & nelx & nely & force & $\beta_{t}$ & $\begin{array}{c}\text { Objective } \\
\text { function }\end{array}$ & $\begin{array}{c}\text { Constraint } \\
\text { function }\end{array}$ \\
\hline DTO & $\mathbf{m}$ & 80 & 16 & $4.0 \times 10^{12}$ & -- & 656 & 0.525 \\
\hline RBTO & $\mathbf{y}$ & 98 & 20 & $4.7 \times 10^{12}$ & 3.8 & 1336 & 0.52 \\
\hline
\end{tabular}

Table 1. DTO and RBTO results for case 1

\section{Case 2: Minimization of fatigue damage subjected to a volume fraction}

The DTO problem is to minimize the maximum value of the fatigue damage subject to a constant fraction of the structural volume. The DTO problem can be written as:

$$
\begin{aligned}
& \min : \max \mathbf{D}(\mathbf{x}) \\
& \text { s.t. }: \frac{V(\mathbf{x})}{V_{0}}=f
\end{aligned}
$$

$f=0.7$ is the proposed volume fraction to be respected. The resulting deterministic topology illustrated in Fig. 4a which verifies an optimal value of the maximum damage, and the required volume fraction $f=0.7$. The RBTO problem integrating the reliability constraint can be written as:

$$
\begin{aligned}
& \min : \max \mathbf{D}(\mathbf{x}) \\
& \text { s.t. }: \frac{V(\mathbf{x})}{V_{0}}=f \\
& \text { and }: \beta(\mathbf{u}) \geq \beta_{t}
\end{aligned}
$$

The reliability-based topology illustrated in Fig. $4 \mathrm{~b}$, gives an optimum value of the maximum damage greater than that the one obtained by DTO, and the volume fraction constraint $f=0.7$ and satisfies the required reliability level.

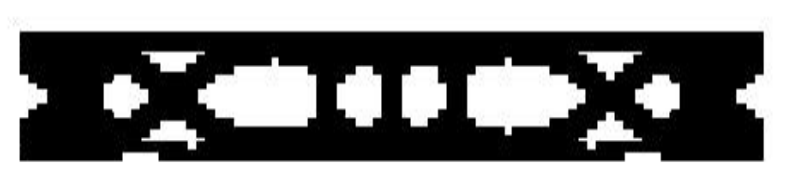

$\boldsymbol{a}$

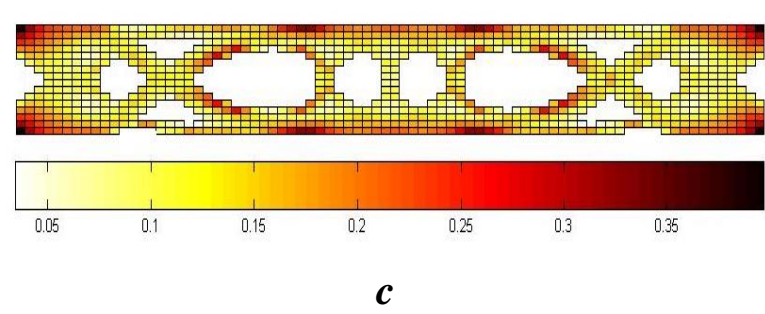

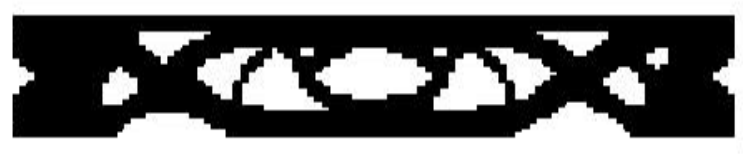

$b$

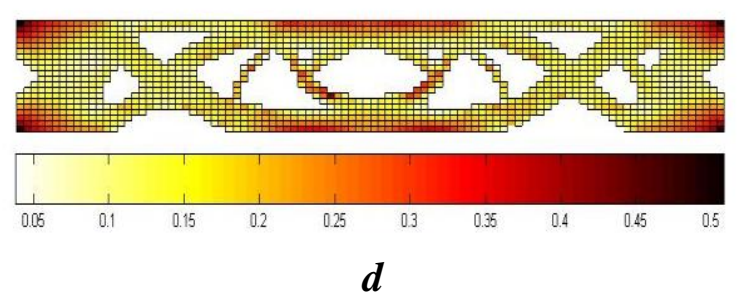

Fig. 4. Topologies and damage distributions: a,c) DTO and b,d) RBTO models

Figs. $4 a, b, c$ and $d$ show the resulting topologies and their damage distributions for the DTO model $(4 a, c)$ and the RBTO model $(4 b, d)$. Table 2 shows the DTO and RBTO results for problems 7 and 8. 


\begin{tabular}{|c|c|c|c|c|c|c|c|}
\hline \multirow{2}{*}{ Model } & \multirow{2}{*}{$\begin{array}{c}\text { Input } \\
\text { vector }\end{array}$} & \multicolumn{4}{|c|}{ Input parameters } & \multicolumn{2}{c|}{ Results } \\
\cline { 3 - 8 } & & nelx & nely & force & $\beta_{t}$ & $\begin{array}{c}\text { Objective } \\
\text { function }\end{array}$ & $\begin{array}{c}\text { Constraint } \\
\text { function }\end{array}$ \\
\hline DTO & $\mathbf{m}$ & 80 & 16 & $4.0 \times 10^{12}$ & -- & 0.3997 & 0.7 \\
\hline RBTO & $\mathbf{y}$ & 98 & 20 & $4.7 \times 10^{12}$ & 3.8 & 0.5114 & 0.7 \\
\hline
\end{tabular}

Table 2. DTO and RBTO results for case 2

\section{Results of DTO and RBTO considering the point of view of "reliability analysis"}

\section{Case 1: Volume minimization subjected to a constraint of fatigue damage}

This case corresponds to a topology optimization problem wherein each element has the same volume $V_{e}$. The DTO problem is to minimize the structural volume, subject to a constraint of fatigue damage. It can be formulated as:

$$
\begin{aligned}
& \min : \operatorname{Volume}(\mathbf{x}) \\
& \text { s.t. }: \max \mathbf{D} \leq \mathrm{D}_{U}
\end{aligned}
$$

where $D_{U}=0.35$ is the maximum fatigue damage (Lambert et al. 2007). The total number of elements is a variable $(n e l=n e l x *$ nely). The resulting deterministic topology illustrated in Fig. 5a, satisfies a minimal volume and the maximum damage $D_{\max }=0.3484 \approx D_{U}$. The limit state used is based on the structural failure criteria. The number of elements in the structure as a random variable satisfies the target reliability index. The RBTO problem pertaining to the integration of the reliability constraint can be formulated as:

$$
\begin{aligned}
& \min : \operatorname{Volume}(\mathbf{x}) \\
& \text { s.t. }: \beta(\mathbf{u}) \geq \beta_{t} \\
& \text { and }: \max \mathbf{D} \leq \mathrm{D}_{U}
\end{aligned}
$$

The resulting reliability-based topology illustrated in Fig. 5b, verifies a minimal volume that is larger than that obtained by DTO, and the maximum damage is less than $D_{U}$. It satisfies the target reliability index.
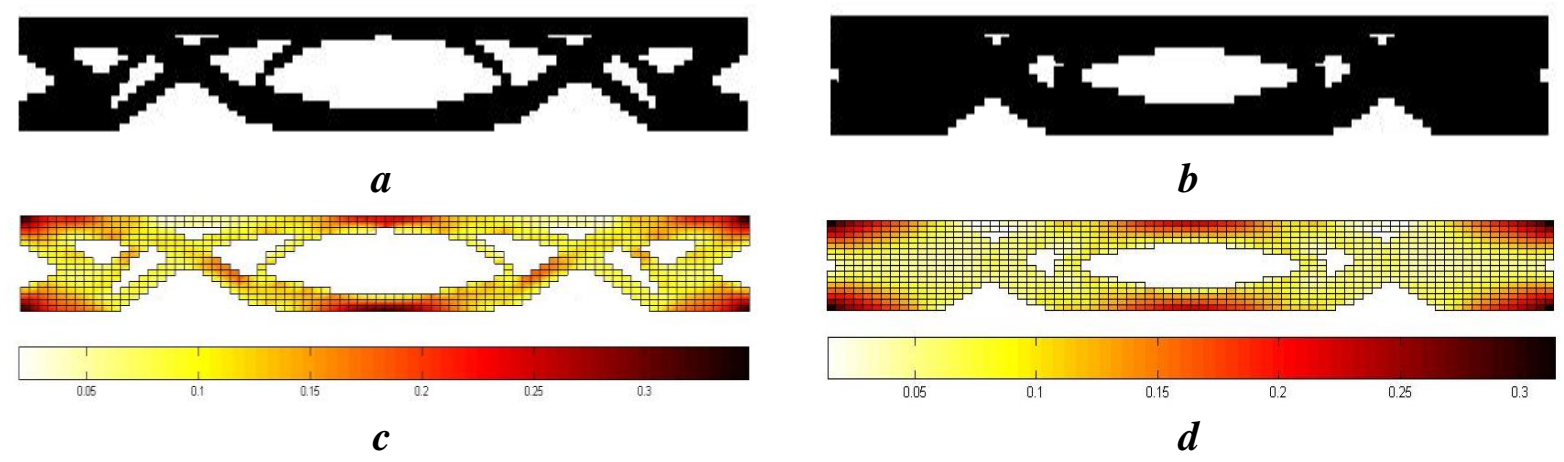

Fig. 5. Topologies and damage distributions with: a, c) DTO and b, d) RBTO models

Figs. 5a, b, c and d show the resulting topologies and their damage distributions for the DTO model ( $5 \mathrm{a}$ and $\mathrm{c}$ ) and for the RBTO model ( $5 \mathrm{~b}$ and d). Table 3 presents the DTO and RBTO results for problems 9 and 10. 


\begin{tabular}{|c|c|c|c|c|c|c|c|}
\hline \multirow{2}{*}{ Model } & \multirow{2}{*}{$\begin{array}{c}\text { Input } \\
\text { vector }\end{array}$} & \multicolumn{4}{|c|}{ Input parameters } & \multicolumn{2}{c|}{ Results } \\
\cline { 3 - 8 } & & nelx & nely & force & $\beta_{t}$ & $\begin{array}{c}\text { Objective } \\
\text { function }\end{array}$ & $\begin{array}{c}\text { Constraint } \\
\text { function }\end{array}$ \\
\hline DTO & $\mathbf{m}$ & 80 & 16 & $4.0 \times 10^{12}$ & -- & 756 & 0.3484 \\
\hline RBTO & $\mathbf{y}$ & 98 & 20 & $4.7 \times 10^{12}$ & 3.8 & 1040 & 0.3160 \\
\hline
\end{tabular}

Table 3. DTO and RBTO results for case 1

\section{Case 2: Minimization of fatigue damage subjected to a volume fraction}

Consider the topology optimization problem, and each element of the mesh has the same volume $V_{e}$. The DTO problem is to minimize the maximum value of the fatigue damage subject to a constraint of volume fraction using the following formulation:

$$
\begin{aligned}
& \min : \max \mathbf{D} \\
& \text { s.t. }: \frac{V(\mathbf{x})}{V_{0}}=f
\end{aligned}
$$

where $f=0.7$ is the volume fraction and the total number of elements is a variable nel. The deterministic topology illustrated in Fig. 6a. It satisfies the optimal value of the maximum damage and the required volume fraction $f=0.7$. The limit state is based on the structure's failure criteria. The number of elements in the structure is a random variable which varies to satisfy the required reliability index. The RBTO problem pertaining to the integration of the reliability constraint can be written as:

$$
\begin{array}{ll}
\min & : \max \mathbf{D} \\
\text { s.t. } & : \beta(u) \geq \beta_{t} \\
\text { and } & : \frac{V(\mathbf{x})}{V_{0}}=f
\end{array}
$$

The resulting reliability-based topology illustrated in Fig. 6b satisfies a minimal volume which is greater than that obtained by DTO, and the maximum damage value is less than $f=0.96>0.7$ in spite of satisfying the required reliability level.

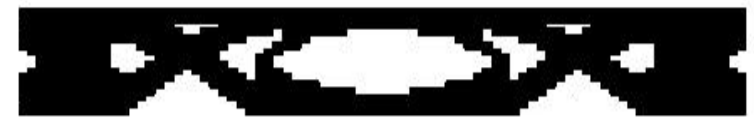

$\boldsymbol{A}$
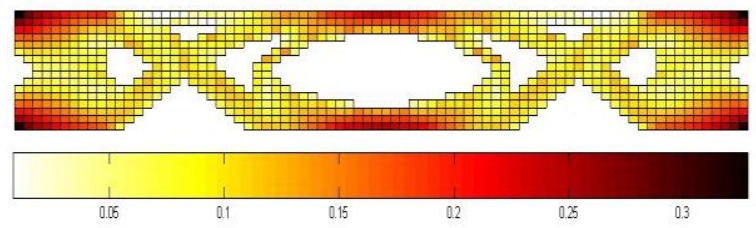

C

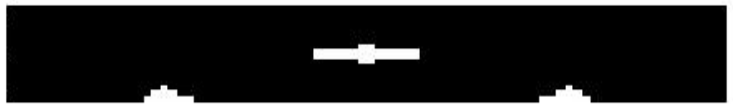

$b$
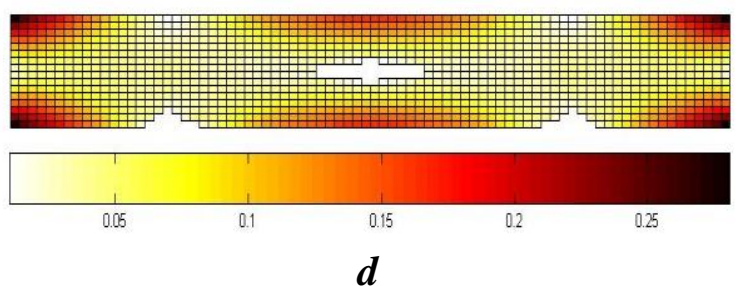

Fig. 6. Topologies and damage distributions: a,c) DTO and b,d) RBTO models 
Figs. 6a, b, c and d show the resulting topologies and their damage distributions for the DTO model (6a and c) and for the RBTO model (6b and d). Table 4 shows the DTO and RBTO results for problems 11 and 12.

\begin{tabular}{|c|c|c|c|c|c|c|c|}
\hline \multirow{2}{*}{ Model } & \multirow{2}{*}{$\begin{array}{c}\text { Input } \\
\text { vector }\end{array}$} & \multicolumn{4}{|c|}{ Input parameters } & \multicolumn{2}{c|}{ Results } \\
\cline { 3 - 8 } & & nelx & nely & force & $\beta_{t}$ & $\begin{array}{c}\text { Objective } \\
\text { function }\end{array}$ & $\begin{array}{c}\text { Constraint } \\
\text { function }\end{array}$ \\
\hline DTO & $\mathbf{m}$ & 80 & 16 & $4.0 \times 10^{12}$ & -- & 0.3304 & 0.7 \\
\hline RBTO & $\mathbf{y}$ & 98 & 20 & $4.7 \times 10^{12}$ & 3.8 & 0.2826 & 0.96 \\
\hline
\end{tabular}

Table 4. DTO and RBTO results for case 2

When comparing the resulting configurations presented in Figs. 3 and 4 and those presented in Figs. 5 and 6, a significant saving of materials can be observed. That leads to consider the DTO and RBTO results considering the point of view 'topology optimization' as an efficient generator of interesting topologies.

\section{Conclusions}

The RBTO model can be integrated into many applications in mechanics because the different developments over the last 20 years provide structures with high performance. This way it allows designers and manufacturers to select a solution which is both economical and reliable. From a point of view 'topology optimization', the reliability constraints are simply introduced to the topology optimization problems. When applying the developed methods from the point of view of topology, the main objective is to generate several solutions for a given data space.

The DTO model can yield a single configuration for a given data space. It has been proven that the RBTO model produces more reliable topologies than those obtained by DTO model for the same weights. The reliability constraints are integrated into the formulation of the topology optimization method considering a linear combination of the random parameters. This approach can be considered as a heuristic strategy which can reduce mass and improve the reliability of the studied structure without increasing the structural weight. The formulation considers the uncertainty linked solely to the geometric dimensions and the applied load.

From the point of view 'reliability analysis', the used limit state function is based on the structural failure criteria. Here, the number of elements in the structure is considered as a number of random variables to satisfy the required level of reliability. The different developments considering the point of view 'reliability analysis' do not generate different configurations when comparing the deterministic and reliability-based topologies. However, our developments with a set of resulting topologies can be widely used in the industrial fields.

\section{References}

Agarwal H. (2004): Reliability Based Design Optimization: Formulations and Methodologies. PhD thesis, University of Notre Dame, 2004.

Bae K.; Wang S. (2002): Reliability-based topology optimization. Proceedings of 9th AIAA/ISSMO Symposium on Multidisciplinary Analysis and Optimization 2002, AIAA 2002-5542.

Bendsøe M.P.; Kikuchi N. (1988): Generating optimal topologies in optimal design using a homogenization method. 
Bendsøe M. P. (1989): Optimal shape design as a material distribution problem. Struct. Optim. 1, 193-202.

Bendsøe M.P.; Sigmund O. (1999): Material interpolations in topology optimization. Arch. Appl. Mech. 69, 635-654.

Eom Y.S.; Yoo K.S.; Park J.Y.; Han S.Y. (2011): Reliability-based topology optimization using a standard response surface method for three-dimensional structures, Journal of Structural and Multidisciplinary Optimization, Vol. 43 (2), pp 287-295, 2011.

Hasofer AM, Lind NC. (1974): An exact and invariant first order reliability format, J. Eng. Mech, ASCE, EM1, 1974; 100:111-121.

Ibrahim M-H.; Kharmanda G.; Charki A. (2015): Reliability-based design optimization for fatigue damage analysis, The International Journal of Advanced Manufacturing Technology, 76: 1021-1030, February 2015.

Jalalpour M.; Tootkaboni M. (2016): An efficient approach to reliability-based topology optimization for continua under material uncertainty, Journal of Structural and Multidisciplinary Optimization, Vol. 53 (4), pp 759-772, 2016.

Jung H-S.; Cho S.; Yang Y-S. (2003): Reliability-based Robust Topology Design Optimization of Nonlinear Structures, WCSMO5, Italy, 2003.

Kharmanda G.; Olhoff N. (2001): Reliability-Based Topology Optimization, Report N: 110, Institute of Mechanical Engineering, Aalborg University, Denmark, December (2001).

Kharmanda G.; Olhoff, N. (2002): Reliability-Based Topology Optimization as a New Strategy to Generate Different Topologies, In: the Nordic Seminar in Computational Methods, (eds. E. Lund, N. Olhoff and J. Stegmsen), Aalborg University, Denmark, October, 18-19 2002.

Kharmanda, G. (2003): CAD \& Optimization of reliable structures, European PhD thesis at Blaise Pascal University, France, 23 June 2003.

Kharmanda G.; Olhoff N.; Mohamed A.; Lemaire M. (2004): Reliability-Based Topology Optimization, Journal of Structural and Multidisciplinary Optimization, Vol. 26, pp 295-307, 2004.

Kharmanda G.; Kourdi N.; Daboul A.; Lambert S.; El Hami A. (2007a): Reliability-based topology optimization as efficient tool for structural designers, 2ème Congrès International Francophone de Mécanique Avancée, CIFMA02, $2^{\text {nd }}$ International Francophone Congress of Advanced Mechanics, IFCAM02, 14-16 May 2007, Aleppo, Syria.

Kharmanda G.; Lambert S.; Kourdi N.; Daboul A.; El-Hami A. (2007b): Reliability-based topology optimization for different engineering applications, International Journal of CAD/CAM , vol 7, 61-69, 2007.

Kharmanda G.; Alkhatab Z. (2011): Nouvelles Méthodes pour la Conception de Topologie, Research Journal of Aleppo University, series of Engineering Sciences, vol. 94, 2011.

Kharmanda G.; Albashi L. (2011): Technologie de l'optimisation de topologie pour augmenter la performance des structures, Research Journal of Aleppo University, series of Engineering Sciences, vol. 97, 2011.

Kharmanda G.; Ibrahim M-H. (2013): Topology-Based Optimum Design Method for Artificially Replaced Cementless Hip Joints, In the: $10^{\text {th }}$ World Congress on Structural and Multidisciplinary Optimization, WCSMO-10, Orlando, Florida, USA, May 19-24, 2013.

Kharmanda G.; Albashi L. (2013): Integration of Topology Optimization into Osteo-Replaced Joint Design, Research Journal of Aleppo University, series of Engineering Sciences, vol. 113, 2013.

Kharmanda G. ; El-Hami A. (2016): Reliability in Biomechanics, ISTE \& Wiley, ISBN: 9781786300249, pp 266, November 2016.

Kharmanda G.; Kharma M-Y. (2016): Evaluating the Effect of Minimizing Screws on Stabilization of Symphysis Mandibular Fracture by 3D Finite Element Analysis, Journal of Maxillofacial and Oral Surgery, 16(2), pp 205-211. DOI: 10.1007/s12663-016-0903-9.

Kharmanda G.; El-Hami A. (2017): Biomechanics: Optimization, Uncertainties and Reliability, ISTE \& Wiley, ISBN: 9781786300256, January 2017.

Kharmanda G.; Antypas I.; Dyachenko A. (2019a): Inverse Optimum Safety Factor Method for Reliability-Based Topology Optimization Applied to Free Vibrated Structures, Journal of Engineering Technologies and Systems, Vol. 29 (1), pp 8-19, 2019. 
Kharmanda, G.; El-Hami, A.; Antypas, I. (2019b): Inverse optimum safety factor method as an effective tool for reliability-based topology optimization: Application on static and dynamic cases, Fifth International Conference on Soft Computing \& Optimisation in Civil, Structural and Environmental Engineering, Riva del Garda, near Lake Garda, Italy16-19 September 2019.

Kharmanda, G.; Antypas I.; Dyachenko A. (2019c): The effect of the reliability index values on the resulting reliability-based topology configurations: Numerical validation by shape optimization, Journal of Engineering Technologies and Systems, Vol. 29 (3), 2019.

Lambert S.; Kharmanda G.; Kadi N.; El-Hami, A. (2007): Integration of system reliability analysis for large-scale fatigue damage problems, 2ème Congrès International Francophone de Mécanique Avancée, CIFMA02, $2^{\text {nd }}$ International Francophone Congress of Advanced Mechanics, IFCAM02, 14-16 May 2007, Aleppo, Syria.

Melchers R-E.; Beck A-T. (2017): Structural Reliability Analysis and Prediction, ISBN:9781119265993, John Wiley \& Sons Ltd., October 2017.

Patel N-M.; Agarwal H.; Tovar A.; Renaud J. (2005): Reliability based topology optimization using the hybrid cellular automaton method. In 1st AIAA Multidisciplinary Design Optimization Specialist Conference, Austin, Texas, April 18-21 2005.

Patel J. and Choi S-K. (2012): Classification approach for reliability-based topology optimization using probabilistic neural networks, Journal of Structural and Multidisciplinary Optimization, Vol. 45 (4), pp 529-543, 2012.

Rozvany, G.I.N. (2000): Problem classes, solution strategies and unified terminology of FE-based topology optimization, In: Rozvany, G.I.N. and Olhoff, N. (eds), Topology optimization of structures and composite continua, 19-35, 2000.

Wang L.; Liu D.; Yang Y.; Wang X.; Qiu Z. (2017): A novel method of non-probabilistic reliability-based topology optimization corresponding to continuum structures with unknown but bounded uncertaintie Computer Methods in Applied Mechanics and Engineering, Vol. 326, pp 573-595, 2017.

Xia L. (2016): Multiscale Structural Topology Optimization, ISTE \& Elsevier, ISBN: 9781785481000, pp 184, April 2016

Zhang W.; Zhu J.; Gao T. (2016): Topology Optimization in Engineering Structure Design, ISTE \& Elsevier, ISBN: 9781785482243, pp 294, October 2016. 